\title{
De la cibernética clásica a la cibercultura: herramientas conceptuales desde donde mirar el mundo cambiante
}

\author{
Begoña Gros \\ Universidad de Barcelona \\ thbgs01ded5.ub.es
}

\section{La cibernética clásica.}

Durante la II Guerra Mundial, a N.Wiener se le encomendó la creación de mecanismos de control para la artillería antiaérea que tuvieran la capacidad de regular su propia trayectoria. Este diseño le condujo al estudio del proceso de regulación de los organismos vivos y lo que Wiener hizo fue extraer los datos que sobre biología se poseían y aplicarlos al diseño de la máquina. Es éste un dato muy relevante pues la comparación funcional entre la mente y la máquina que posteriormente incorporó la psicología cognitiva y la inteligencia artificial encuentran aquí sus orígenes. Pero también es importante porque hasta aquel momento no había habido ninguna relación entre los estudios biológicos y el diseño de máquinas, aspecto presente en la actualidad tanto en el diseño del hardware como del software. Hecho que demuestra en la actualidad las investigaciones sobre el desarrollo de redes neuronales como base para la creación de los sistemas operativos de las máquinas y la utilización de la nanotecnología (Kurzweil, 1999)

Al principio, el interés de la cibernética se situó en el diseño de máquinas. Sin embargo, el propio Wiener amplió dicho objetivo y lo extendió al entendimiento del funcionamiento de sistemas humanos y sociales (Wiener, 1969).

\section{La noción de circularidad.}

La cibernética introduce la idea de circularidad a través del concepto de retroalimentación o feedback. El feedback se define como la capacidad de respuesta para el mantenimiento de un estado de equilibrio. El feedback es pues un mecanismo que conduce a la regulación de un sistema. La regulación se produce siempre tras la ruptura de equilibrio. Es decir, cuando el estado ideal del sistema no coincide con su estado actual. En este caso, el sistema reacciona produciéndose una nueva búsqueda de equilibrio.

La utilización del concepto de feedback rompe la idea de causalidad tradicional en la que los efectos se encadenan de forma lineal. Por el contrario, el concepto de feedback conduce a la descripción de procesos circulares. Un bucle de retroalimentación es una disposición circular de elementos conectados, en la que una causa inicial se propaga alrededor de los eslabones sucesivos del bucle, de tal modo que cada elemento tiene efecto sobre el siguiente, hasta que el último elemento vuelve a retroalimentar el efecto sobre el primero que inicio el proceso. La causalidad circular es siempre procesual.

La idea de circularidad desarrollada por Wiener se centra en el feedback negativo. La reacción del sistema tiene un efecto contrario al del elemento perturbador $\mathrm{y}$, por consiguiente, éste busca recuperar el equilibrio a través del mantenimiento del estado ideal. En definitiva, es este un feedback de tipo "conservador" ya que la meta o estado ideal del sistema se mantiene constante. Wiener reconocía además a la retroalimentación 
como el mecanismo esencial de la homeostasis, la autorregulación que permite a los organismos vivos mantenerse en un estado de equilibrio dinámico.

En 1968, Maruyama introdujo el concepto de feedback positivo que, a diferencia del negativo, amplifica la desviación. Este tipo de retroacción conduce a una desviación cada vez mayor respecto al estado ideal. Por este motivo, el feedback positivo podría llevar a la destrucción del sistema. No obstante, puede también convertirse en un mecanismo de creación de nuevos sistemas o de reestructuración de los ya existentes. La utilización de este concepto ha permitido explicar la evolución de los sistemas sociales y humanos en los cuales se complementan y entrecruzan los dos tipos de retroacciones.

\section{El concepto de información.}

La información es para la teoría cibernética un elemento fundamental para la organización del sistema. Lo que el enlace circular de los componentes retroalimenta no es sólo materia y energía, sino que hay allí un proceso informacional y organizacional. Determinar el significado del término información, a pesar de su clara importancia, sigue siendo muy problemático. Como afirma Morin "no podemos decir casi nada acerca de ella, pero tampoco podemos prescindir de ella" (1994, p.47).

El concepto de información utilizado por la cibernética tiene su origen en la teoría comunicativa de Shannon y Weaver que trataba de la transmisión de mensajes y, actualmente está integrada en la teoría de la comunicación. Esta noción de información está basada en la medida de la información a través de unidades elementales de información o bits (binary digits), muy utilizados en la actualidad en la informática y las telecomunicaciones. A esta dimensión de la información, la cibernética le añade un sentido organizacional. De hecho, un "programa" portador de información lo que hace es comunicar un mensaje a una máquina que computa un cierto número de operaciones.

Tal y como afirma Capra, "Wiener enfatizó también el hecho de que el mensaje codificado es esencialmente un patrón organizador, y esbozando la analogía entre tales patrones de comunicación y las pautas de organización en los organismos, sentó las bases de la visión de los organismos vivos en términos de patrones" (1998, p.83).

En definitiva, la aportación ofrecida por la perspectiva cibernética de mayor interés es la concepción de la información como elemento de organización.

\section{Patrón/Estructura.}

La teoría cibernética y la teoría de sistemas se desarrollan a un mismo tiempo y las conexiones entre ambas son muy importantes. La mayor parte de los autores sistémicos utilizan para el análisis de los sistemas el aparato conceptual de la teoría cibernética y, por otra parte, la mayor parte de las cibernéticas tienen una concepción sistémica de la realidad. Por este motivo, es realmente difícil separar ambas visiones y, de hecho, a partir de este momento me voy a referir frecuentemente a aspectos de la teoría de sistemas para poder comprender mejor la propia cibernética.

Desde el punto de vista sistémico, el aspecto fundamental de cualquier estudio basado en los sistemas vivos radica en la síntesis entre el estudio de la substancia o estructura del sistema y el estudio de su forma o patrón. En el estudio de la estructura lo que hacemos es fijarnos en la medida de los elementos que conforman el sistema. Los patrones, en cambio, nos muestran la forma del sistema, su cartografía. En este sentido, la estructura puede ser medida, analizada cuantitativamente mientras que al patrón sólo nos podemos aproximar desde un punto de vista cualitativo. 
La ciencia clásica se centra en el estudio de los elementos que componen la realidad. Los estructuralistas miran a través de las interrelaciones que se establecen entre los elementos que componen una determinada realidad. La perspectiva sistémica es contextual y por consiguiente, no sólo busca el análisis de la estructura sino también la cualidad del sistema estudiado.

El patrón de estudio básico de la teoría cibernética está basado en la comunicación y el control basado en la idea de circularidad. Cualquier sistema vivo se desarrolla a través de procesos circulares generados por de comunicación y controlados desde el propio sistema.

\section{Evolución y proceso.}

En un sistema vivo, la evolución se basa en un proceso entre los vínculos establecidos por el patrón y la estructura. "Un sistema vivo es a la vez abierto y cerrado: abierto estructuralmente, pero cerrado organizativamente. La materia y la energía fluyen a través de él, pero el sistema mantiene una forma estable y lo hace de manera autónoma, a través de su autoorganización" (Capra, 1999, p.182).

El empeño de buena parte de los cibernéticos ha consistido en descubrír los patrones de organización comunes a todos los seres vivos. Este es el caso de Bateson, Maturana y Varela quienes consideran que la materia y la mente no son dos categorías separadas sino que representan dos aspectos diferentes del mismo fenómeno de la vida. La mente es un proceso que se identifica con el proceso de la vida. El empeño de todos estos autores ha sido conseguir desarrollar teorías integradoras del hombre en que las diversas dimensiones vitales: percepción, emoción, acción, lenguaje, etc., queden incluidas en una sola red cognitiva explicativa de la mente humana.

\section{La visión holística del mundo.}

La contribución más importante de la teoría general de sistemas de Bertalanffy es la demostración de que los sistemas vivos no pueden ser comprendidos desde el análisis. Las propiedades de las partes, de los elementos, no tienen propiedades intrínsecas y, en consecuencia, sólo pueden entenderse desde el contexto del todo. Por tanto, el pensamiento sistémico es un pensamiento contextual. Sin embargo, la mirada de los sistémicos a menudo se ha vuelto excesivamente objetual. Desde mi punto de vista, el pensamiento sistémico debe ser un pensamiento contextual ya que el proceso y los cambios marcan la dinámica fundamental de los sistemas. Sin embrago, el empeño de la mayoría de los autores es un empeño de cartógrafo. Tratan de marcar los sistemas existentes, sus fronteras y acaban siendo teorías donde la vida no está presente.

En este sentido, considero muy importante la crítica efectuada por Wilber (1995) quien cree que la teoría de sistemas se centra en dibujar los aspectos externos del sistema social. Por ello, dibuja tan sólo una parte del mundo, dejando de lado los aspectos culturales, conductuales, individuales y sociales.

La perspectiva holística debe ser más integradora y, en cierta medida, las teorías de la complejidad están ayudando a ello proporcionando nuevos conceptos que permitan mirar a los sistemas desde un enfoque menos racionalista.

\section{La cibernética de la complejidad.}

En 1958, Heinz von Foerster efectúa una revisión crítica de la teoría de Wiener, concluyendo que la cibernética que éste había desarrollado introducía cambios importantes pero no suponía una ruptura epistemológica ya que se seguía aplicando el 
modelo de la ciencia clásica por el cual el observador está fuera del objeto (del sistema, en este caso) y es capaz de estudiarlo con objetividad. Von Foerster consideró que la cibernética debía ir más allá y afrontar un nuevo modelo epistemológico en el cual el observador formara parte del sistema estipulando sus propios objetivos, su propio papel dentro del mismo. A partir de ese instante se efectúa una distinción entre la cibernética clásica o cibernética de primer orden y la cibernética de segundo orden, denominada también como teoría de la complejidad. La pregunta que se hace la cibernética de primer orden podemos formularla de la siguiente forma: ¿dónde están los enlaces circulares en este sistema?. Mientras que la cuestión que se plantea la cibernética de segundo orden es: ¿cómo generamos nosotros este sistema a través de la noción de circularidad?.

La cibernética de segundo orden ha sido desarrollada por autores como von Foerster, Glasersfeld, Bateson, Prigogine, Maturana, Morin y Varela, entre otros, y, en la actualidad muchos de sus principios han quedado también plasmados en la teoría de los sistemas complejos o teoría de la complejidad.

La teoría cibernética de segundo orden introduce una serie de cambio de mirada de gran interés que sintetizaré en tres aspectos: la construcción de la realidad, la autoorganización de los sistemas y el concepto de entropía.

\subsection{La construcción de la realidad.}

En realidad, la cibernética de segundo orden es una teoría de un carácter fuertemente epistemológico. Se cuestiona la concepción de la ciencia clásica en la cual existe una clara separación entre el sujeto y el objeto de investigación.

Desde un punto de vista epistemológico, la cibernética de primer orden se inscribe dentro de la corriente realista u objetivista que considera que el conocimiento se refiere a una realidad estable, objetiva, existente independientemente a que ésta sea conocida por el hombre. Por el contrario, la epistemología desarrollada por la cibernética de segundo orden se enraíza en la filosofía kantiana y considera que "el mundo de la experiencia, ya se trate de la experiencia cotidiana o de la experiencia de laboratorio, constituye la piedra de toque para nuestras ideas." (Glasersfeld, 1988, 23). En este sentido, el conocimiento no implica una correspondencia con la realidad.

Glasersfeld (1988) utiliza las palabras inglesas "match" (corresponder) y "fit" (encajar) para explicar las diferencias entre ambas posiciones. El realismo considera que existe una correspondencia entre el conocimiento y la realidad mientras que este autor sostiene que el conocimiento supone tan sólo un encaje. De este modo, diferentes conocimientos, significados, interpretaciones y experiencias pueden encajar en una misma realidad. En definitiva, tal y como afirma Maturana, "el observador se hace en la observación y cuando el ser humano que es el observador muere, el observador y la observación llega a su fin" $(1994,158)$.

La perspectiva desarrollada por la cibernética de segundo orden es, en definitiva, una perspectiva constructivista. De hecho, autores como von Foerster o Glasersfeld se presentan en la actualidad como representantes del constructivismo radical (Glasersfeld 1988; 1994; Von Foerster 1991).

Este tipo de constructivismo es muy similar al desarrollado por Piaget. Desde esta perspectiva cibernética, la construcción es el resultado de la autorregulación del sistema. Para que haya construcción del conocimiento debe haber también un proceso de reflexión. La función de la cognición es adaptativa y sirve para organizar el mundo experiencial del sujeto y no para describir una verdad o realidad ontológica objetiva. El conocimiento es pues como un mapa de senderos de acciones y pensamientos que en el 
momento de la experiencia se han convertido en viables.

El concepto de viabilidad fue establecido por Glasersfeld (1988) y hace referencia a aquellos conceptos que son útiles para la supervivencia. Según este autor, en la praxis, el juicio de una teoría se apoya única y exclusivamente en el hecho de que hasta ahora no ha fracasado. El conocimiento tiene que ser viable, adecuarse a nuestros propósitos. De las teorías se mantienen aquellos conceptos que resultan útiles para la supervivencia. En definitiva, el conocimiento equivale a una función de supervivencia y no a una descripción del mundo exterior. Lo que interesa es que los conocimientos que construyo encajen lo suficiente como para asegurar su viabilidad.

\subsection{Los sistemas se autoorganizan.}

Como ya he señalado previamente, los cibernéticos distinguieron entre el patrón de organización de un sistema y su estructura física. En este sentido, consideraron que la comunicación y el control eran suficientes para explicar la organización de los sistemas. La cibernética de segundo orden introduce el concepto de autoorganización y la idea de complejidad para completar esta visión.

El concepto de autoorganización se basa en el reconocimiento de la red como patrón general de la vida que Maturana y Varela denominaron autopoiesis. La autopoiesis se basa en la idea de que existen una serie de relaciones entre componentes que deben regenerarse continuamente para mantener su organización y mantener un equilibrio dinámico.

En este contexto, los procesos de circularidad ya no se basan sólo en los mecanismos de retroalimentación negativa sino que la retroalimentación positiva muestra cómo en determinados momentos la búsqueda del equilibrio conlleva a una mayor desviación y, por ello, el sistema acaba reestructurándose $\mathrm{o}$ creando nuevos patrones de funcionamiento.

La diferencia fundamental entre el primer concepto de autoorganización de la cibernética y los modelos posteriores estriba en que éstos incluyen la creación de nuevas estructuras y nuevos modelos de comportamiento que operan lejos de procesos de equilibrio, y por tanto, no siguen procesos lineales.

\subsection{El desorden que crea orden.}

La teoría cibernética de segundo orden se fundamenta en una revisión de la segunda ley de la termodinámica realizada por Prigogine. La segunda ley de la termodinámica establece que en un sistema cerrado hay perdidas constantes de energía que introducen cada vez un mayor desequilibrio, un estado de entropía, de desorden. Sin negar la veracidad de esta ley, Prigogine consideró que los sistemas vivos son sistemas abiertos en los que la inestabilidad no destruye al sistema sino que produce nuevo orden y crea una mayor complejidad. En definitiva, en una situación de mucho desequilibrio aparecen elementos de indeterminación (no predecibles) que introducen un orden y una mayor complejidad.

Un sistema humano, un sistema social, no es un sistema en equilibrio. Por el contrario, constantemente se producen perturbaciones, desviaciones que fuerzan a una constante reorganización y ajuste. En este sentido, el orden y el desorden "cooperan" para la organización del sistema. El desorden es necesario para la producción del orden. Esta relación dialéctica forma parte de la complejidad de los sistemas.

\section{La cibercultura.}


Durante muchos años, el empleo del término "cibernética" ha estado reservado a los estudiosos o especialistas en este ámbito de trabajo. Era muy poco probable su uso en revistas o en la prensa diaria. Sin embargo este hecho cambia totalmente a partir de los años 90. La palabra cibernética se utiliza, en primer lugar, asociada al uso de la tecnología informática y, posteriormente, al uso de las redes de comunicación. Sin embargo, en este uso más masivo, el propio término se ve mutilado apareciendo nuevas uniones tales como ciberespacio, cibercultura, ciberfeminismo, cibersexo, etc. No hablaré aquí de todos los "cibers" generados sino que me centraré en el uso del concepto de cibercultura al que tomaré como sinónimo de ciberespacio usándolos indistintamente.

La cibercultura hace referencia a todos los cambios de índole cultural que se están generando como consecuencia de la utilización de la informática como medio de información y comunicación. La cibercultura tiene la peculiaridad de estar desprovista de centro, de líneas directrices, de entidades ligadas a un tiempo y un espacio. En este sentido, como dice P. Virilio (1997), no se habla de cibercultura por casualidad ya que las autopistas de la información están unidas a un fenómeno de feedback, de retroacción constante, la interactividad marca el aspecto fundamental del sistema social actual.

Ahora bien, la cibernética que fundamenta el funcionamiento de la red no se basa únicamente en modelos de circularidad tal y como señala Virilio, sino que, en mi opinión, el modelo del ciberespacio se aproxima mucho más al modelo de complejidad elaborado por la cibernética de segundo orden. Por ello, la mirada cibernética nos proporciona una descripción de los sistemas virtuales que se están generando en la sociedad actual.

La cibercultura, el ciberespacio está fundamentado en los siguientes aspectos:

\subsection{Sociedad-Red.}

Con el desarrollo de las redes de comunicación, especialmente de Internet que crece día a día de forma exponencial, se dibuja una sociedad con una visión circular del mundo que se autoenlaza y se autorreproduce. Si un sistema puede ser visto en forma circular, estamos ante un sistema en que es muy difícil diferenciar sus formas y sus límites. Desde el punto de vista cibernético, podemos considerar la sociedad como un sistema orgánico viviente basado en la comunicación y la información que se generan y se introducen según su funcionalidad. No hay mensajes fuera de contexto, separados de una comunidad activa. Todos los mensajes forman parte de una comunidad activa, están llenos de vida y mueren cuando dejan de tener sentido dentro del contexto en que han sido generados.

En definitiva, "esta concepción permite tratar las redes como circulaciones sin comienzo ni fin, en la medida en que sus "empalmes" son múltiples, y sus encaminamientos, complejos. Se la recogerá bajo la forma de una visión circular del mundo y sus envolturas sucesivas; visión de un interior orgánico que se autoenlaza para una reproducción asimismo "auto"” (Sfez,1995). No sólo es un sistema abierto sino que es muy difícil diferenciar su forma y sus limites. El orden y el desorden se generan a través de la interacción y la participación activa de los usuarios que son a la vez productores y consumidores del propio sistema. No hay un centro y por consiguiente, el sistema está constituido por redes de redes. De hecho, tal y como afirma Lèvy (1998), el espacio no es desorden sino expresión de la diversidad de lo humano.

Según Lèvy (1998), la cibercultura es el universal sin totalidad. El ciberespacio no genera una cultura de lo universal porque está en todas partes, sino porque su forma o su idea implican el derecho al conjunto de los seres humanos. Dice un anuncio de una empresa de telecomunicaciones "la comunicación es un derecho, no un privilegio". Esta empresa ha captado perfectamente el sentido de lo universal sin totalidad. 


\subsection{Comunicación-Conectividad.}

"Conectarse, estar conectado" son expresiones frecuentes. La conexión, como mencionaba previamente, es un bien en sí mismo, y por tanto un derecho. Estar conectado representa estar en el mundo, formar parte del sistema, lo que te permite a su vez ser creador de nuevos sistemas. La conectividad es condición necesaria para la comunicación a través de la red. Las formas comunicativas están transformándose dentro de la red a través de la creación de las comunidades virtuales.

Las comunidades virtuales se construyen sobre afinidades, intereses y conocimientos independientemente de la proximidad geográfica. Son comunidades muy diversas en su composición y también en su estructura y forma comunicativa. De hecho, existen reglas y normas no escritas que se van generando en la red y que se aplican a medios distintos de comunicación. Por ejemplo, en un chat no se escribe con mayúsculas porque indican enfado. En un Forum, puede echarse a un miembro por enviar mensajes que no se centren en el tema a debate, o por anunciar un producto comercial.

Las formas comunicativas son muy variadas y, al contrario de lo que muchos autores parecen opinar, no creo que sean excluyentes de otras formas de comunicación. El uso del correo electrónico no está eliminando el uso del teléfono sino todo lo contrario.

\subsection{La Información.}

Como señala E. Havelock (1996), el paso de la oralidad a la escritura en Grecia marcó un cambio fundamental en el desarrollo del pensamiento occidental. La escritura supuso un cambio no sólo del medio de comunicación sino de la forma de conciencia. La escritura sirvió para fijar el conocimiento, las reglas y normas sociales. "No es la creatividad, sea ésta lo que fuera, sino el recuerdo y la memoria los que contienen la clave de nuestra existencia civilizada " (Havelock, 1996, p.104). El lenguaje hablado queda fijado en un vocabulario y en un orden fijador.

La manera de usar los sentidos y nuestra forma de pensar está relacionadas, en la transición de la oralidad a la escritura. La oralidad enfatizaba el oído y la boca, la escritura enfatiza el ojo y la mano. Debido a este cambio, la civilización occidental ha utilizado el lenguaje escrito como fuente de desarrollo del conocimiento y del propio pensamiento. La racionalidad es explícitada a través del propio lenguaje que organiza y fija el conocimiento. Por este motivo, McLuhan considera que toda la civilización occidental ha estado centrada en el desarrollo del hemisferio izquierdo del cerebro [1] y ello ha contribuido al desarrollo del razonamiento cuantitativo. En cambio, la cultura oriental ha mantenido el espacio acústico proyectado hacia el hemisferio derecho desarrollando un pensamiento más cualitativo, más holista.

Los medios de comunicación y las tecnologías poseen una estructura fundamentalmente lingüística, ya no hay un predominio de la escritura sino de lo visual. La información generada en la red está más próxima a la cultura de la oralidad que a la cultura de la escritura aunque con cambios importantes. La información no se transmite de generación a generación sino que es volátil, se crea y desaparece cuando deja de ser funcional para la comunidad que la ha generado. Todo el mundo puede producir información por lo que no hay diferencia entre productor y consumidor.

Los cambios cognitivos no son rápidos. Hemos tardado muchos siglos en conocer la influencia de la escritura en el pensamiento y no es posible saber cómo las tecnologías de la información y la comunicación van a alterar nuestra cognición. Lo que sí resulta claro, es que necesariamente va a haber una alteración que ya estamos viviendo pero que todavía no podemos analizar con exactitud. 


\subsection{Velocidad}

En una novela, Milan Kundera recoge, en una frase, el sentido de la situación actual: "Hay un vínculo secreto entre la lentitud y la memoria, entre la velocidad y el olvido...

En la matemática existencial, esta experiencia adquiere la forma de dos ecuaciones elementales: el grado de lentitud es directamente proporcional a la intensidad de la memoria; el grado de la velocidad es directamente proporcional a la intensidad del olvido.” (Milan Kundera, La lentitud.)

La velocidad ha ido aumentando con el desarrollo de la tecnología pero nunca hasta ahora el crecimiento había sido tan rápido. Por ello, en la sociedad informacional hay una ruptura del ritmo y de los ciclos vitales. Tal y como afirma Castells "el tiempo atemporal se da cuando las características de un contexto determinado, a saber, el paradigma informacional y la sociedad-red, provocan una perturbación sistémica en el orden secuencial de los fenómenos realizados en ese contexto" $(1998$, p. 499)

\section{Los medios que masajean la educación}

"Todos los medios nos vapulean minuciosamente. Son tan penetrantes en sus consecuencias personales, políticas, económicas, estéticas, psicológicas, morales, éticas y sociales, que no deja parte alguna de nuestra persona intacta, inalterada, sin modificar. El medio es el masaje".

(M.McLuhan, 1967)

Como señaló el propio McLuhan, toda nueva tecnología amplifica, exterioriza y modifica muchas funciones cognitivas. En la sociedad informacional, hay claras modificaciones en la memoria (bases de datos, hiperdocumentos, ficheros de todo tipo), imaginación (simulaciones), percepción (realidades virtuales, telepresencia) y la propia comunicación.

Los medios no sólo nos masajean sino que masajean a la educación, se introducen en nuestras vidas y, de pronto, nos damos cuenta de su influencia. No acabo de entender muy bien las razones, pero los profesionales de la educación se ocupan poco de los medios. Los critican, eso sí, con mucha frecuencia. Los contenidos de los medios no son apropiados, los niños pasan demasiado tiempo delante de la televisión, del ordenador, de la consola,... No leen porque hay informática. No salen a jugar porque hay ordenadores. Los padres miran a sus hijos, los dejan delante de la televisión. Ven la programación infantil y no hay problema (eso piensan), Los educadores les advierten: vea la televisión con sus hijos, introduzca el espíritu crítico, analice los mensajes. Pocos lo hacen. Los padres miran los ordenadores, ven a sus hijos absortos delante de la pantalla. ¿Cómo pueden pasar tantas horas concentrados delante del ordenador?, controlan el tiempo que pasan, no son capaces de hacer nada más. Los educadores les advierten: no todo lo que hay en Internet es positivo para sus hijos, cuidado con los juegos que le compra. DESCONCIERTO TOTAL.

El problema es que los profesionales de la educación también están desconcertados. Hay que ser flexible, integrar medios, diseñar nuevos modos de formación, nuevas formas comunicativas. ¿Por dónde empezamos?. Este es el reto y, a la vez, un camino que los profesionales de la educación no pueden descuidar. Nuestros alumnos de hoy son muy diferentes a los de hace diez años, no podemos enfocar el proceso de enseñanzaaprendizaje de la misma forma $\mathrm{y}$, es fundamental, crear profesionales capaces de contribuir a un buen desarrollo de las tecnologías de la información y la comunicación para que la cibercultura sea de verdad, una cultura. 
CAPRA,F (1998). La trama de la vida. Barcelona: Anagrama.

CASTELLS, M (1997). La era de la información. Madrid: Alianza. Vol 1.

FOERSTER,H (1991). Las semillas de la cibernética. Barcelona: Gedisa.

GLASERSFELD, E (1994) "Despedida de la objetividad", pp.19-31, en Watzlawick, PFrieg,P (eds.) El ojo del observador. Barcelona: Gedisa.

GLASERSFELD, E.(1988). Introducción al constructivismo radical, en Watzlawick,P (ed.) La realidad Inventada .Barcelona: Gedisa. pp.20-37

HAVELOK, E. (1996). La musa aprende a escribir. Barcelona: Paidós.

KERCKHOVE,D (1999). La piel de la cultura. Barcelona: Gedisa.

KURZWEIL, R (1999). La era de las máquinas espirituales. Barcelona: Planeta.

LEVY, P. (1 998). La cibercultura, el segon diluvi? Barcelona: UOC/Proa.

MARUYAMA,M (1968). "The Second Cybernetics: Deviation-Amplifying Mutual Causal Processes", en Buckley,W (ed) Modern Systems Research for the Behavioral Scienciest, Chicago: Aldine.

MARUYAMA, H.(1994). "La ciencia y la vida cotidiana: la ontología de las explicaciones científicas", en Watzlawick, P-Frieg,P (eds.) El ojo del observador. Barcelona: Gedisa. pp. 157-195

MCLUHAN, E-ZINGRONE,F (Comp). McLuhan. Escritos esenciales. Barcelona: Paidós.

MCLUHAN, M (1997). El medio es el masaje. Un inventario de efectos. Barcelona: Paidós.

MCLUHAN, M.-POWERS, B (1993). La aldea global. Barcelona: Gedisa.

SFEZ,L (1995). Crítica de la comunicación. Buenos Aires: Amorrortu.

VILAR,S (1997). La nueva racionalidad. Barcelona: Kairós.

WIENER,N. (1960) Cibernética y Sociedad. Buenos Aires:Editorial Sudamericana.

WILBER,K (1995). Sexo, ecología y espiritualidad. Madrid: Gaia.

WILBER,K (1996). Breve historia de todas las cosas. Barcelona: Kairós.

\section{NOTAS}

[1] En el cerebro izquierdo predominan los aspectos racionales (pensamiento lógicomatemático, secuencial, analítico, etc.). En cambio en el cerebro derecho predomina los aspectos emocionales, intuitivos, la visión holística. 
(C) Ediciones Universidad de Salamanca. 